\title{
Open partial nephrectomy - Case report and review of literature on stage T2 tumors
}

\begin{abstract}
Partial nephrectomy is currently the gold standard treatment for renal tumors of up to $4 \mathrm{~cm}$ at their widest area. It has also been gaining support for use on even larger tumors around the world. We report a case of a 74-year-old patient with an $11 \mathrm{~cm}$ lesion on the right kidney associated with left renal atrophy, which illustrates the emerging worldwide trend of performing nephron-sparing surgery on patients with T2 tumors. The patient underwent a partial nephrectomy, which then had favorable clinical evolution during the follow-up period. Additionally, we present a brief review of the literature addressing such topics as surgical indications, complications, the advantages and disadvantages of performing partial versus radical nephrectomy, and assessment of survival rates (in total as well as those for patients free from disease). Currently, nephron-sparing surgery has emerged as a satisfactory and oncologically safe option for larger tumors, a practice that was not advocated until recently.
\end{abstract}

Keywords: partial nephrectomy, renal cell carcinoma, radical nephrectomy, T2 tumors, urinomas
Volume 6 Issue 5 - 2018

\author{
Saulo da Cunha Recuero, ', 'Túlio Agresta,' \\ Rodrigo Piltcher da Silva, ${ }^{3}$ Matheus \\ Bernardon Morillos, ${ }^{3}$ Sabino Junior ${ }^{3}$ \\ 'Urologist and postgraduate of University of São Paulo (USP), \\ Brazil \\ ${ }^{2}$ Urologist of Sancta Maggiore (Prevent Senior), Brazil \\ ${ }^{3}$ Medical student, The Federal University of Pelotas (UFPEL), \\ Brazil
}

Correspondence: Dr. Saulo da Cunha Recuero, University of São Paulo, Brazil,Tel (I I) 949849889,

Email saulorecuero@hotmail.com

Received: June 27, 2018| Published: October 02, 2018

\section{Introduction}

Renal cancer is the third most common type of urological cancer, behind only prostate and bladder cancer (Figure 01 ). It affects around 300,000 people per year worldwide, according to the International Agency of Research on Cancer, and mainly affects people in Asia and Europe. ${ }^{1}$ In Brazil, there is no concrete data on its incidence, according to the latest publication of the National Cancer Institute (INCA) from 2018; ${ }^{2}$ however, in the United States of America, the American Cancer Society predicts that in 2018, 65,340 new cases of renal cancer will occur with approximately 15,000 associated deaths. The incidence of this neoplasm in the USA will be of 16 people per 100,000 inhabitants, with a higher prevalence in men. ${ }^{3}$

Renal cell carcinoma (RCC) accounts for about $90 \%$ of malignant neoplasms of the kidney. ${ }^{4}$ In recent years, with improvements in diagnostic imaging techniques and increases in their rate of usage, growth in the incidence of this neoplasm has been reported. ${ }^{5}$ Most cases are diagnosed in asymptomatic patients incidentally during routine examinations. ${ }^{6}$ The classic triad of flank pain, hematuria, and palpable abdominal mass are present in less than $10 \%$ of patients, and among the symptomatic, $30 \%$ already experience metastatic disease at diagnosis. ${ }^{4,6}$

There are four main histological patterns of RCC: clear-cell carcinoma, papillary carcinoma, chromophobe carcinoma, and collecting duct carcinoma. ${ }^{4}$ The most prevalent is a clear-cell carcinoma, which accounts for $80-90 \%$ of all cases. ${ }^{4,6}$ Staging of these tumors is performed through the TNM system, which takes into account the tumor size, affected lymph nodes, and distant metastases. ${ }^{4}$

Radical nephrectomy was considered the standard treatment for RCC more than 50 years ago, supported by the work of Robson et al. ${ }^{7}$ In recent years, partial nephrectomy (NP) has been gaining ground for localized tumors, which until then had usually been performed only for T1a tumors (up to $4 \mathrm{~cm}$ ) and T1b tumors (up to $7 \mathrm{~cm}$ ). ${ }^{4,8}$ Currently, with the advancement of surgical techniques, there is evidence suggesting the use of NP can also be effective for more advanced tumors, such as stage T2 tumors. ${ }^{4,6,8,9}$ With this information in mind, we will address a case of nephron-sparing nephrectomy (NPN) for a $\mathrm{T} 2 \mathrm{~b}$ tumor, discussing the indications and therapeutic effects.

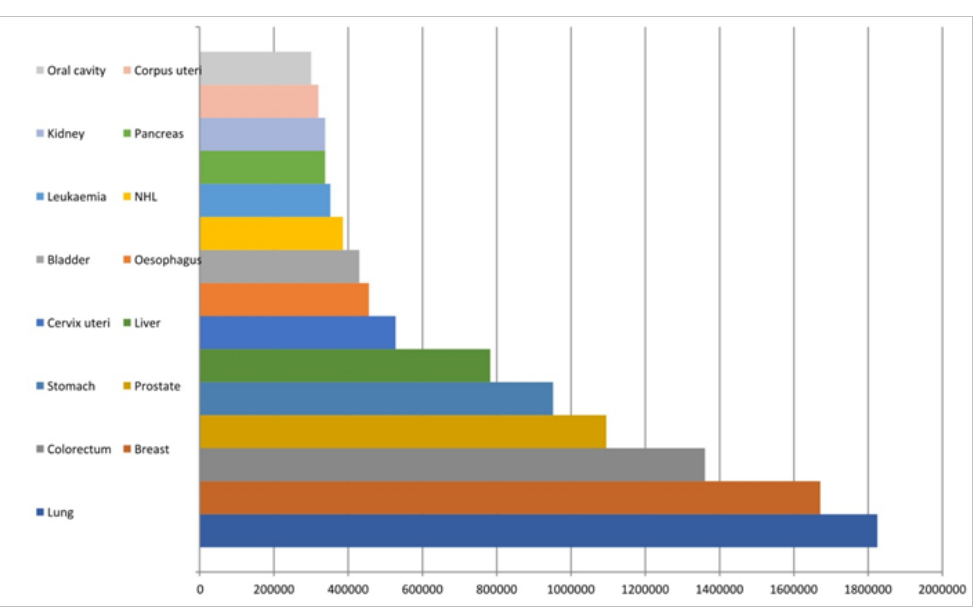

Figure I Incidence of cancer cases for both sexes globally in 2012 - adapted from GLOBOCAN 20 I 2 - International Agency of Research on Cancer 20 I8. 


\section{Case report}

A 74-year-old female patient was referred to the urology department of a private hospital for a right kidney mass associated with left renal atrophy. She reported that symptoms began one year prior, starting with intermittent hematuria and lower back pain for three months. During an abdominal physical examination, the mass was palpated in the right renal store, with no other changes present. Upon laboratory analysis, it presented the following: hemoglobin $(\mathrm{Hb}) 13.0 \mathrm{~g} / \mathrm{dl}$, Urea (Ur) $50 \mathrm{mg} / \mathrm{dl}$, Creatinine $(\mathrm{Cr}) 1.2 \mathrm{mg} / \mathrm{dl}$, and a glomerular filtration rate (GFR - MDRD) 46,68ml/min/1,73m2. A computed tomography (CT) scan of the abdomen revealed a hypervascular expansive formation of $10 \times 11.2 \times 6.8 \mathrm{~cm}$, with exophytic and endophytic components, less than $1 \mathrm{~cm}$ away from the collecting system, centered on the anterolateral aspect of the kidney, exceeding the limit of the lower polar line by more than 50\% (Figure 2), RENAL Nephrometry Score is $10 \mathrm{x}$.

The patient had a history of hypertension, diabetes, hyperthyroidism, and ischemic stroke (IS) seventeen years prior, which resulted in motor sequelae. The patient had previously undergone the following surgeries: appendectomy, hysterectomy, and a surgery for the removal of benign breast nodules.
It was decided that a right partial open nephrectomy would be performed due to the volume of the tumor. The access route was through an anterior lombotomy without peritoneal cavity violation, through which the NPN was deemed as technically viable. Hot ischemia was performed for tumor resection with the opening of the collecting pathway and posterior raffia of the same with absorbable wire, hemostatic control, and a vascular clamp, which was removed within 16 minutes of ischemia. The intraoperative bleeding was approximately 300 milliliters $(\mathrm{ml})$ and the surgical procedure took around three and a half hours. The patient showed good clinical evolution, but worsened renal function, in the immediate postoperative period. There was subsequent stabilization with hospital discharge on the fifth postoperative day.

The specimen weighed 640 grams and anatomopathological analysis with immunohistochemistry confirmed renal cell carcinoma of the chromophobe type, with the renal capsule free of neoplastic involvement and the absence of invasion of Gerota's fascia. Postoperative follow-up was performed through laboratory analysis (Figure 3), physical examination, and imaging examination (Figure 4) within 90 days of surgery without evidence of relapse of the disease and the patient maintaining preserved global renal function.

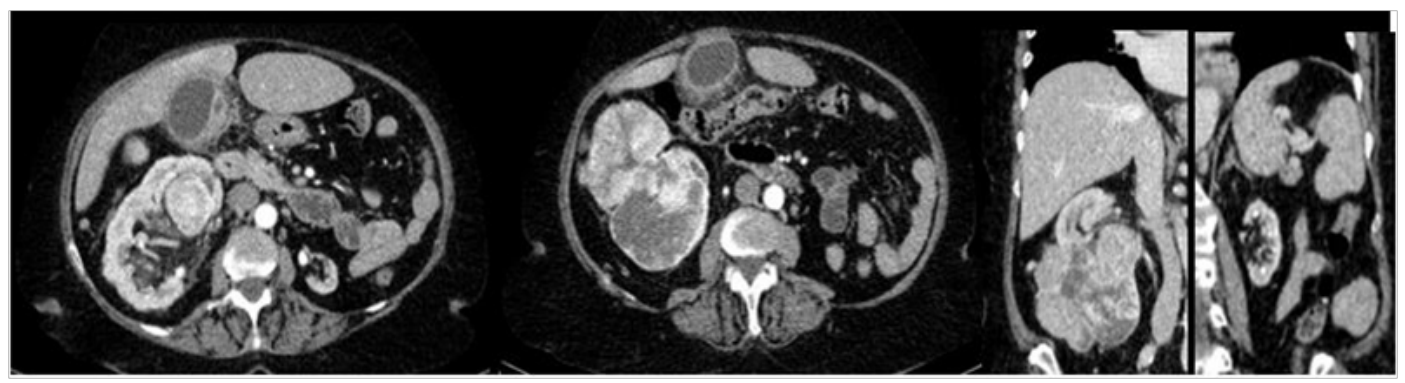

Figure 2 CT scan of the abdomen with contrast showing right tumor mass with the atrophic left kidney.

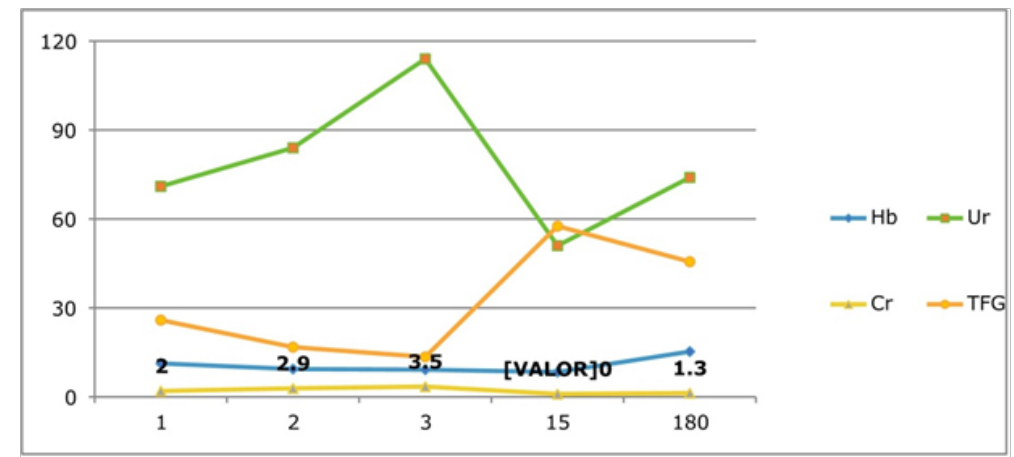

Figure 3 Laboratory follow-up in the postoperative period. $\mathrm{Hb}=$ hemoglobin in $\mathrm{g} / \mathrm{dl}, \mathrm{Ur}=\mathrm{urea}$ in $\mathrm{g} / \mathrm{dl}, \mathrm{Cr}=\mathrm{creatinine}$ in $\mathrm{g} / \mathrm{dl}, \mathrm{GFR}=\mathrm{glomerular}$ filtration rate (MDRD).

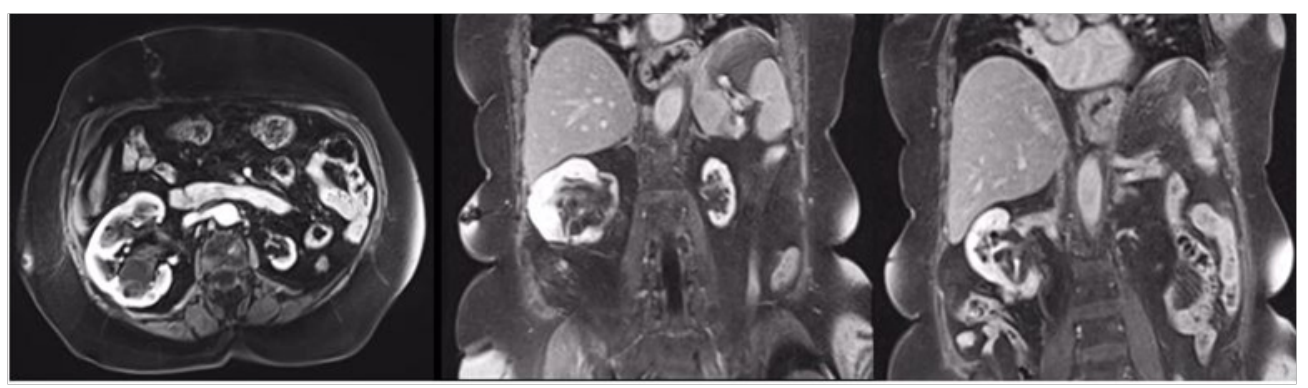

Figure 4 Magnetic resonance imaging of the abdomen with contrast without evidence of recurrence with vicarious right kidney postoperative follow-up. 


\section{Discussion}

Among renal tumors, those classified as T1 have partial nephrectomy as the therapeutic option of choice, which is already accepted globally. ${ }^{7}$ On the other hand, in T2 tumors, the role of partial nephrectomy in elective cases is still a matter of debate, although this therapeutic option is gaining increasing popularity. ${ }^{10,11}$ In these cases, the surgical choice depends on factors such as previous comorbidities, surgical risk, and the anatomical complexity of the tumor. This is evaluated by means of nephrometry, for which RENAL ${ }^{12}$ and PADUA $^{13}$ are the most commonly used systems for predicting the degree of tumor complexity and the rate of conversion to radical nephrectomy by assessing the time to ischemia, surgical margin, and complications. ${ }^{14-16}$ However, it is now known that tumor size should not be considered an independent contraindication to this procedure as previously believed. ${ }^{8,9}$ In addition, there are situations where there is an imperative indication for NPN, such as in chronic renal failure (CRF), single-kidney renal tumor, and bilateral renal tumor. ${ }^{8,10}$

Nephron-sparing surgery has obtained similar oncological results as radical nephrectomy for renal masses larger than $7 \mathrm{~cm}$, with the benefit of a lower incidence of chronic kidney disease and its complications, such as the need for hemodialysis, renal transplant, anemia, osteoporosis, and cardiovascular disease. ${ }^{17,18}$ For example, Huang et al. ${ }^{19}$ demonstrated that patients undergoing NP have a lower risk of developing chronic kidney disease when compared to those submitted to NR $(80.0 \%$ vs $35.0 \%, \mathrm{p}<0.001) .{ }^{19}$ The meta-analysis of Mir et al. ${ }^{11}$ when comparing more than 21 case-control studies involving more than 11 thousand patients submitted to partial versus radical nephrectomy for $\mathrm{T} 1 \mathrm{~b}$ and $\mathrm{T} 2$ tumors, showed low rates of tumor recurrence, cancer-specific mortality, and overall mortality in cases submitted to nephron-sparing nephrectomy. ${ }^{1}$

Surgical complications such as urinary fistula and bleeding were more common in partial nephrectomy but had no relevant impact. ${ }^{8,10}$ Mir et al. ${ }^{11}$ observed that the difference in blood loss during NP, when compared to NR, was only $100 \mathrm{ml} .{ }^{11}$ Fistulas were treated with drainage and rarely needed reoperation. Urinomas had a good response to a double J catheter implantation..$^{8,10}$ On the other hand, there were higher rates of infection of the operative wound, sepsis, and ileus in the NR. Beside this, there was a significant decrease in glomerular function and $\mathrm{CRF}$, which were the main long-term complications of this procedure. ${ }^{10}$

Some studies compared video laparoscopic surgery (VLP) with open surgery for partial nephrectomy, finding similar results for both overall survival rates and disease-free survival rates. ${ }^{4,5}$ In VLP there was a lower rate of bleeding, but a longer period of hot ischemia and a more pronounced GFR in the immediate postoperative period, with no difference in the longer term. ${ }^{20}$ Thus, the technique used to perform the NP does not influence the final results. The surgeon must choose the technique according to the available equipment and his experience with each method. ${ }^{5}$

\section{Conclusion}

The therapeutic approach to renal tumors has changed in recent years. With these changes, partial nephrectomy, once considered an option only for T1 tumors, has been shown to be a good alternative for the treatment of larger and more complex masses.
The greater surgical risk attributed to nephron-sparing surgery is acceptable since it presents no significant risk of mortality and has lower morbidity rates. Still, it is characterized by very satisfactory oncological results and a better overall survival when compared to patients undergoing radical nephrectomy.

\section{Acknowledgements}

None.

\section{Conflict of interest}

Author declares that there is no conflict of interest.

\section{References}

1. Cancer fact sheets. World Health Organization (WHO). GLOBOCAN. 2012.

2. Estimate: 2018 Incidence of Cancer in Brazil. Ministry of Health, National Cancer Institute, José Alencar Gomes da Silva (INCA), Brazil. 2018.

3. Cancer Statistics Center. USA: American Cancer Society. 2018.

4. Ljungberg B, Bensalah $\mathrm{K}$, Canfield $\mathrm{S}$, et al. EAU guidelines on renal cell carcinoma: 2014 update. Eur Urol. 2015;67(5):913-924.

5. Lane BR, Gill IS. 7-year oncological outcomes after laparoscopic and open partial nephrectomy. J Urol. 2010;183(2):473-479.

6. Ljungberg B, Cowan NC, Hanbury DC, et al. EAU guidelines on renal cell carcinoma: the 2010 update. Eur Urol. 2010;58(3):398-406.

7. Robson CJ. Radical nephrectomy for renal cell carcinoma. J Urol. 1963;89:37-42.

8. Kopp RP, Liss MA, Mehrazin R, et al. Analysis of Renal Functional Outcomes After Radical or Partial Nephrectomy for Renal Masses $>/=7$ cm Using the RENAL Score. Urology. 2015;86(2):312-319.

9. Karellas ME, O Brien MF, Jang TL, et al. Partial nephrectomy for selected renal cortical tumours of $>/=7 \mathrm{~cm}$. BJU Int. 2010;106(10):1484-1487.

10. Breau RH, Crispen PL, Jimenez RE, et al. Outcome of stage T2 or greater renal cell cancer treated with partial nephrectomy. $J$ Urol. 2010;183(3):903-908

11. Mir MC, Derweesh I, Porpiglia F, et al. Partial Nephrectomy Versus Radical Nephrectomy for Clinical T1b and T2 Renal Tumors: A Systematic Review and Meta-analysis of Comparative Studies. Eur Urol. 2017;71(4):606-617.

12. Kutikov A, Uzzo RG. The R.E.N.A.L. nephrometry score: a comprehensive standardized system for quantitating renal tumor size, location and depth. J Urol. 2009; 182(3):844-853.

13. Ficarra V, Novara G, Secco S, et al. Preoperative aspects and dimensions used for an anatomical (PADUA) classification of renal tumours in patients who are candidates for nephron-sparing surgery. Eur Urol. 2009;56(5):786-793

14. Hsieh PF, Wang YD, Huang CP, et al. A Mathematical Method to Calculate Tumor Contact Surface Area: An Effective Parameter to Predict Renal Function after Partial Nephrectomy. J Urol. 2016;196(1):33-40.

15. Leslie S, Gill IS, de Castro Abreu AL, et al. Renal tumor contact surface area: a novel parameter for predicting complexity and outcomes of partial nephrectomy. Eur Urol. 2014;66(5):884-893.

16. Borgmann H, Reiss AK, Kurosch M, et al. R.E.N.A.L. Score Outperforms PADUA Score, C-Index and DAP Score for Outcome Prediction of Nephron Sparing Surgery in a Selected Cohort. J Urol. 2016;196(3):664-671. 
17. Woldrich J, Mehrazin R, Bazzi WM, et al. Comparison of rates and risk factors for development of anaemia and erythropoiesis-stimulating agent utilization after radical or partial nephrectomy. BJU Int. 2012;109(7):1019-1025.

18. Bagrodia A, Mehrazin R, Bazzi WM, et al. Comparison of rates and risk factors for development of osteoporosis and fractures after radical or partial nephrectomy. Urology. 2011;78(3):614-619.
19. Huang WC, Levey AS, Serio AM, et al. Chronic kidney disease after nephrectomy in patients with renal cortical tumours: a retrospective cohort study. Lancet Oncol. 2006;7(9):735-740.

20. Gill IS, Kavoussi LR, Lane BR, et al. Comparison of 1,800 laparoscopic and open partial nephrectomies for single renal tumors. $J$ Urol. 2007;178(1):41-46. 